\title{
Dynamics of Selective Recall in an Associative Memory Model with One-to-Many Associations
}

\author{
Masaki Kawamura, Masato Okada, and Yuzo Hirai
}

\begin{abstract}
The dynamics of selective recall in an associative memory model are analyzed in the scenario of one-to-many association. One-to-many association is one of the most important characteristics of our memory system because a homophone, for example, associates with more than one word and each word can have several meanings. The present model, which can deal with one-to-many association, consists of a heteroassociative network and an autoassociative network. In the heteroassociative network, a mixture of associative items in one-to-many association is recalled by a key item. In the autoassociative network, the selective recall of one of the associative items is examined by providing a seed of a target item either to the heteroassociative network (Model 1) or to the autoassociative network (Model 2). We show by both simulation studies and theoretical analysis that the critical similarity of Model 2 is not sensitive to the change in the dimension ratio of key vectors to associative vectors, and it has smaller critical similarity (correlation between the seed and the target item) than Model 1 for a large initial overlap. On the other hand, we show that Model 1 has smaller critical similarity for a small initial overlap. We also show that unreachable equilibrium states exist in the proposed model. There is a critical loading rate $\alpha_{r}$ where the reachable equilibrium states are disappeared. Above the critical loading rate $\alpha_{r}$, which is smaller than the storage capacity $\alpha_{c}$, all equilibrium states are stable, but cannot be reached.
\end{abstract}

Index Terms - Associative memory, one-to-many association, selective recall, statistical neurodynamics.

\section{INTRODUCTION}

$\mathbf{O}$ NE-TO-MANY association is one of the most important characteristics in our associative memory system because a homophone, for example, can associate with more than one word and each word can have several meanings. To recall the most appropriate item in some situation, we usually use context. Let us consider two homophones (words), "sun" and "son," for example. Since the same pronunciation is associated with the two words, we cannot decide which word has been pronounced by just listening to the pronunciation. However, if a context word such as "family" is given, "son" can be recalled easily. The psychological literature suggests that there are two stages in the process of resolving lexical ambiguities [1]-[3]. The first is a sense activation stage, in which all associated meanings are activated, and the second

Manuscript received April 23, 1998; revised December 11, 1998 and February 2, 1999.

M. Kawamura is with 3E205, Doctoral Program in Engineering, University of Tsukuba, Tsukuba-shi, 305-8573 Japan.

M. Okada is with the KAWATO Dynamic Brain Project, ERATO, Japan Science and Technology Corporation (JST), 2-2 Hikaridai, Seika-Cho, Sorakugun, Kyoto 619-0288, Japan.

Y. Hirai is with the Institute of Information Sciences and Electronics, University of Tsukuba, Tsukuba-shi, 305-8573 Japan.

Publisher Item Identifier S 1045-9227(99)03874-6. is a sense selection stage, in which the most suitable meaning is selected using the context and the rest of the meanings are suppressed.

Hirai proposed the associative memory model HASP, which can deal with one-to-many associations [4]. It consists of a heteroassociative network and a mutually inhibitory network that is cascaded to the heteroassociative network. In the heteroassociative network all the items associated with key inputs are recalled, and in the mutually inhibitory network one of the recalled items is selected through competition. The mutually inhibitory network stores a summation of the autocorrelation matrix of associative items in the form of reduced inhibitory connections. Since the HASP can deal with context as one of the key inputs, one of the associative items associated with these will be selected. The HASP was also applied to constraint satisfaction problems and modeling cognitive processes such as the processes of addition and of learning counting [5]-[7].

The HASP consists of two networks, while Amit [8] proposed a model which consists of one network with both crosscorrelation and autocorrelation in a synaptic weight. Although the Amit model deals with one-to-one associations, it would be interesting to extend the Amit model to be able to treat the one-to-many associations. Guyon et al. [9] proposed the model which can recall complex sequences with branches, that is, it can deals with one-to-many associations. The model, however, cannot select the items dynamically.

We propose a HASP-type associative memory model. The structure of the proposed model is qualitatively equivalent to that of the HASP, but for the sake of simplicity the mutually inhibitory network has been replaced with an autoassociative network. The autoassociative network can memorize the associative items in equilibrium states, and the associative item which is most similar to the initial state can be recalled. Many statistical mechanical approaches [10]-[13] have been introduced to analyze the model, since Hopfield [14] gave the spin system analogy. These approaches consider the equilibrium state of the network and can analyze the storage capacity. The dynamical behavior, however, cannot be discussed in these approaches. Amari and Maginu [15] proposed the statistical neurodynamics, which can explain the dynamical behavior of the networks qualitatively. Okada [16], moreover, expands the Amari-Maginu theory into higher-order theory, which can explain the dynamical behavior almost quantitatively. This paper analyzes the recall process selecting a target item, which is one of the associative items that is recalled in the heteroassociative network. In the associative memory with one-to-many associations, the dynamical behavior must be taken into account, since the associative item to be recalled 


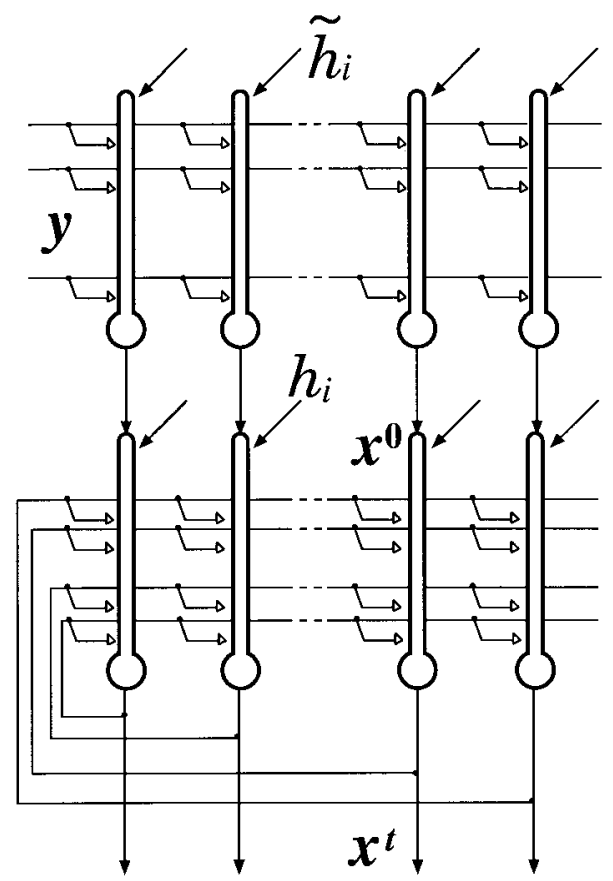

Fig. 1. A schematic diagram of the present model. The upper network operates as the heteroassociative memory, while the lower network operates as the autoassociative memory.

may be dynamically changed according as the context. The proposed model, therefore, is analyzed by the statistical neurodynamics.

In the proposed model, we assume that a cue, which is derived from context, is supplied as an external input. The supplied cue comes from a context word and may be similar to the target item. In this paper, we will not discuss the detailed mechanism to generate the cue. A seed of the target item is supplied either to the heteroassociative network or to the autoassociative network. A temporal evolution of overlap (direction cosine) between the target item and the output of the present model is analyzed by changing the similarity (correlation) between the seed and the target item.

This paper is structured as follows. Section II describes the model we used and introduces and defines all the parameters in analysis. Section III presents the theoretical analysis of recall dynamics and corresponding simulation results. Section IV concludes this paper.

\section{PROposed MODEL}

Fig. 1 shows the structure of the present model. It consists of a heteroassociative network and an autoassociative network. We introduced external input which provides the seed of the target item either to the heteroassociative network (denoted by $\tilde{h}_{i}$ ) or the autoassociative network (denoted by $h_{i}$ ).

In the following, we define the time when a key input, denoted by $\boldsymbol{y}$, is presented to the heteroassociative network as $t=-1$, and define the time when the output from the heteroassociative network, denoted by $x^{0}$, is fed to the autoassociative network as $t=0$.

\section{A. Heteroassociative Network}

Let us assume that each of the $p$ key vectors $\boldsymbol{\eta}^{\mu}=\left(\eta_{i}^{\mu}\right), \mu=$ $1,2, \cdots, p$ is associated with $k^{\mu}$ associative vectors $\boldsymbol{\xi}^{\mu, \kappa}=$ $\left(\xi_{i}^{\mu, \kappa}\right), \kappa=1,2, \cdots, k^{\mu}$, that is, the key items are associated with different number of associative items. The dimensions of the key vectors and the associative vectors are assumed to be $M$ and $N$, respectively. Each component of the vectors $\boldsymbol{\eta}^{\mu}$ and $\xi^{\mu, \kappa}$ is assumed to be an independent random variable which takes a value of either +1 or -1 according to the following probabilities:

$$
\operatorname{Prob}\left[\eta_{i}^{\mu}= \pm 1\right]=\frac{1}{2}
$$

and

$$
\operatorname{Prob}\left[\xi_{i}^{\mu, \kappa}= \pm 1\right]=\frac{1}{2}
$$

In this paper, we have mainly considered a case where the following conditions are satisfied: 1) Let $M=\beta N . \beta \sim O(1)$ holds as $N \rightarrow \infty$. 2) Let $\sum_{\mu=1}^{p} k^{\mu}=\alpha N$, where $p \sim O(N)$ and $k^{\mu} \sim O(1)$ hold and $\alpha$ is loading rate.

The output of the heteroassociative network is given by

$$
x_{i}^{0}=\operatorname{sgn}\left(\sum_{j=1}^{M} \tilde{J}_{i j} y_{j}+\tilde{h}_{i}\right)
$$

where $\tilde{J}_{i j}$ is a synaptic weight from the $j$ th component of the key input $\boldsymbol{y}$ to the $i$ th neuron of the network. The external input $\widetilde{h}_{i}$ was introduced to provide the seed to recall one of the associative vectors recalled by the key input. It will be described in detail in Section II-C. The synaptic weight $\tilde{J}_{i j}$ is determined by correlation learning:

$$
\tilde{J}_{i j}=\frac{1}{N} \sum_{\mu=1}^{p} \sum_{\kappa=1}^{k^{\mu}} \xi_{i}^{\mu, \kappa} \eta_{j}^{\mu}
$$

In the following, we will analyze a case where the associative vector $\xi^{1,1}$ is recalled by the key vector $\boldsymbol{\eta}^{1}$, that is, the target vector is $\boldsymbol{\xi}^{1,1}$. In order to take incomplete key inputs into account, the overlap or direction cosine between the key input $\boldsymbol{y}$ and the key vector $\boldsymbol{\eta}^{1}$ is defined as

$$
\tilde{m}=\frac{1}{M} \sum_{j=1}^{M} \eta_{j}^{1} y_{j}
$$

which is termed initial overlap. Then, (3) can be expressed in terms of the initial overlap $\tilde{m}$ as

$$
x_{i}^{0}=\operatorname{sgn}\left(\sum_{\kappa=1}^{k^{1}} \beta \tilde{m} \xi_{i}^{1, \kappa}+\tilde{z}_{i}+\tilde{h}_{i}\right)
$$

where $\tilde{z}_{i}$ is crosstalk noise at time $t=-1$ which is given by

$$
\tilde{z}_{i} \equiv \frac{1}{N} \sum_{j=1}^{M} \sum_{\mu \neq 1}^{p} \sum_{\kappa=1}^{k^{\mu}} \xi_{i}^{\mu, \kappa} \eta_{j}^{\mu} y_{j}
$$




\section{B. Autoassociative Network}

The autoassociative network consists of $N$ neurons that are connected with each other. The state $x_{i}^{t+1}$ of the $i$ th neuron for $t \geq 0$ is defined by

$$
x_{i}^{t+1}=\operatorname{sgn}\left(\sum_{j \neq i}^{N} J_{i j} x_{j}^{t}+h_{i} \delta_{t, 0}\right)
$$

where $J_{i j}$ is a synaptic weight from the $j$ th neuron to the $i$ th neuron, and $h_{i}$ is an external input which will be described in detail in Section II-C. $\delta_{t, 0}$ is Kronecker's delta and is defined as $\delta_{t, 0}=1$ when $t=0$, and $\delta_{t, 0}=0$ when $t \neq 0$. Since $h_{i}$ is supplied only at time $t=0$, it has no effect on the stability of equilibrium states. The synaptic weight $J_{i j}$ is given by correlation learning:

$$
J_{i j}=\frac{1}{N} \sum_{\mu=1}^{p} \sum_{\kappa=1}^{k^{\mu}} \xi_{i}^{\mu, \kappa} \xi_{j}^{\mu, \kappa} .
$$

We define the overlaps between the state $\boldsymbol{x}^{t}=\left(x_{i}^{t}\right)$ appearing in the recall process at time $t$ and the associative vectors $\xi^{1, \kappa}$ as

$$
m_{t}^{\kappa}=\frac{1}{N} \sum_{i=1}^{N} \xi_{i}^{1, \kappa} x_{i}^{t} .
$$

Then, (8) can be expressed in terms of the overlaps $m_{t}^{\kappa}$ as

$$
x_{i}^{t+1}=\operatorname{sgn}\left(\sum_{\kappa=1}^{k^{1}} m_{t}^{\kappa} \xi_{i}^{1, \kappa}+z_{i}^{t}+h_{i} \delta_{t, 0}\right)
$$

where $z_{i}^{t}$ is crosstalk noise at time $t \geq 0$ which is given by

$$
z_{i}^{t} \equiv \frac{1}{N} \sum_{j \neq i}^{N} \sum_{\mu \neq 1}^{p} \sum_{\kappa=1}^{k^{\mu}} \xi_{i}^{\mu, \kappa} \xi_{j}^{\mu, \kappa} x_{j}^{t} .
$$

\section{External Input}

Here we explain why the external inputs, $\tilde{h}_{i}$ and $h_{i}$, were introduced in (3) and (8), respectively. For simplicity, let us assume $M=N$, namely, $\beta=1.0$ and $p \sim O(1)$. Then the loading rate becomes $\alpha=0$ because $k^{\mu} \sim O(1)$. In this paper, we will only concentrate on one-to-three associations $\left(k^{\mu}=3\right)$ for the sake of simplicity. The present analysis can easily be extended to general cases with arbitrary $k^{\mu}$. Let us assume that the key input $\boldsymbol{y}$ which is identical with the key vector $\eta^{1}(\tilde{m}=1.0)$ is supplied to the heteroassociative network. The output $x_{i}^{0}$ from the heteroassociative network at time $t=0$ becomes the initial state of the autoassociative network and is given by

$$
x_{i}^{0}=\operatorname{sgn}\left(\xi_{i}^{1,1}+\xi_{i}^{1,2}+\xi_{i}^{1,3}+\tilde{h}_{i}\right) .
$$

If no external input $\tilde{h}_{i}$ is injected, the state $x_{i}^{0}$ becomes a mixture state $\zeta^{1}=\left(\zeta_{i}^{1}\right)$, each component of which is given by

$$
\zeta_{i}^{1}=\operatorname{sgn}\left(\xi_{i}^{1,1}+\xi_{i}^{1,2}+\xi_{i}^{1,3}\right) .
$$

Since the output $x_{i}^{0}$ is the mixture state $\zeta^{1}$, the state of the autoassociative network at time $t=1$ changes to

$$
x_{i}^{1}=\operatorname{sgn}\left(\frac{1}{2} \xi_{i}^{1,1}+\frac{1}{2} \xi_{i}^{1,2}+\frac{1}{2} \xi_{i}^{1,3}+h_{i}\right)
$$

where the $1 / 2$ 's denote overlaps $m_{0}^{\kappa}, \kappa=1,2,3$ between the state $\boldsymbol{x}^{0}=\boldsymbol{\zeta}^{1}$ and the associative vectors $\xi^{1, \kappa}$. If no external input $h_{i}$ is injected, the state $\boldsymbol{x}^{t}$ for every $t \geq 1$ will not change and it becomes an equilibrium state. In order to select the target vector $\xi_{i}^{1,1}$, external input is introduced as the seed, which is similar to $\xi_{i}^{1,1}$. By providing external input, the state will move close to the target vector. Based on these considerations recall dynamics is analyzed for the two typical models following.

Model 1: $\tilde{h}_{i}$ is injected into the heteroassociative network at time $t=-1$

$$
\operatorname{Prob}\left[\tilde{h}_{i}= \pm 1\right]=\frac{1 \pm a \xi_{i}^{1,1}}{2}, \quad h_{i}=0 .
$$

Model 2: $h_{i}$ is injected into the autoassociative network at time $t=0$

$$
\tilde{h}_{i}=0, \quad \operatorname{Prob}\left[h_{i}= \pm 1\right]=\frac{1 \pm a \xi_{i}^{1,1}}{2} .
$$

From (16) and (17), the correlations between $\xi_{i}^{1,1}$ and the external inputs, $\tilde{h}_{i}$ and $h_{i}$, are given by

$$
E\left[\xi_{i}^{1,1} \tilde{h}_{i}\right]=E\left[\xi_{i}^{1,1} h_{i}\right]=a .
$$

The correlation " $a$ " in (18) is termed as similarity between the target vector $\xi^{1,1}$ and external input, and it varies in the range $0 \leq a \leq 1$. If $a=0$, the external input, $\tilde{h}_{i}$ or $h_{i}$, takes a value of \pm 1 at random and it is statistically independent of $\xi_{i}^{1,1}$. If $a=1, \tilde{h}_{i}$ or $h_{i}$ is equal to $\xi_{i}^{1,1}$. Then, the networks may recall $\xi^{1,1}$ when $a=1$, but they will probably fail to recall when $a=0$. Therefore, we can expect that there is a critical similarity $a_{c}$ and the target vector can be recalled above it. We suppose that the critical similarity of Model 1 differs from that of Model 2. The main purpose of the present paper is to evaluate the two models using critical similarity.

\section{RESUlTS AND DISCUSSION}

\section{A. Distribution of Crosstalk Noise}

We will show that both the heteroassociative and the autoassociative network need to be analyzed together. Here we assume that the external input is $\tilde{h}_{i}=0$. The distribution of the crosstalk noise at time $t=0$ will be obtained, when the initial state is supplied into the autoassociative network. First, let us consider that the key input $\hat{\boldsymbol{x}}^{0}$, which correlates with $\xi^{1,1}$ but without the other associative vectors $\xi^{\mu, \kappa}$, is supplied into the autoassociative network. Here the crosstalk noise $\hat{z}_{i}^{0}$

$$
\hat{z}_{i}^{0}=\frac{1}{N} \sum_{j \neq i}^{N} \sum_{\mu \neq 1}^{p} \sum_{\kappa=1}^{k^{\mu}} \xi_{i}^{\mu, \kappa} \xi_{j}^{\mu, \kappa} \hat{x}_{j}^{0}
$$

which is the contribution from the other associative vectors, is normally distributed with mean 0 and variance $\alpha$ [15]. Fig. 2 shows the distribution for the crosstalk noise $\hat{z}_{i}^{0}$ with a loading 


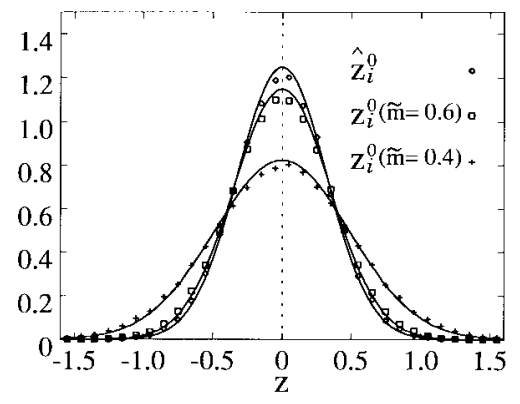

Fig. 2. The distributions of crosstalk noise, where $M=N=500$ and $\alpha=0.1$. $\hat{z}_{i}^{0}$ indicates the distribution for the autoassociative network and $z_{i}^{0}$ 's indicate the distributions for the present model, where the values of initial overlap are $\tilde{m}=0.4$ and 0.6 .

rate of $\alpha=0.1$, obtained by theoretical analysis and simulation studies, where $N=500$.

Next, let us consider that the key input $\boldsymbol{y}$ with the initial overlap $\tilde{m}$ is supplied into the heteroassociative network and that the output of the heteroassociative network, $x^{\mathrm{O}}$, is supplied into the autoassociative network. Here the initial state $x^{0}$ of the autoassociative network is correlated with all associative vectors. In order to evaluate the distribution of crosstalk noise, this correlation must be taken into account. The macroscopic state equations of the present model, therefore, were derived using statistical neurodynamics [15], [16]. The equations used in theoretical analysis are briefly described in the Appendix. Fig. 2 shows the distributions for the crosstalk noise $z_{i}^{0}$ of (12) obtained by the macroscopic state equations, where the values of the initial overlap are $\tilde{m}=0.4$ and 0.6 . From (26), the crosstalk noise $z_{i}^{0}$ is normally distributed with mean 0 and variance $\sigma_{0}^{2}$.

Each solid line denotes the results obtained by theoretical analysis, while corresponding simulation results are represented by points $(M=N=500$ ). Both results agree with each other. Fig. 2 shows that the crosstalk noise $z_{i}^{0}$ strongly depends on the initial overlap $\tilde{m}$. That is why the two networks need to be analyzed together. In analyzing the recall process, we showed that the correlations between the state $x^{0}$ and the associative vectors $\xi^{\mu, \kappa}$ must be taken into account. The important point is that the variance of the crosstalk noise $z_{i}^{0}$ is not only larger than the variance of $\hat{z}_{i}^{0}$ but it is also dependent on the initial overlap $\tilde{m}$. The distribution for the crosstalk noise $z_{i}^{0}$ when $\tilde{m}=0.6$ is more similar to that of $\hat{z}_{i}^{0}$ than that of $z_{i}^{0}$ when $\tilde{m}=0.4$. Fig. 3 shows the variance of $\hat{z}_{i}^{0}$ and $z_{i}^{0}$ in the case of $\alpha=0.1$. We can see that the variance of $z_{i}^{0}$ is strongly dependent on the initial overlap $\tilde{m}$. It also indicates that the distribution of $\hat{z}_{i}^{0}$ is a good approximation of that of $z_{i}^{0}$ when $\tilde{m} \rightarrow 1$. Therefore, regarding the absolute storage capacity, we can safely analyze the HASP by dividing it into the two networks [17]. The term absolute means the probability that any associative vector $\xi^{\mu, \kappa}$ is stable will converge to 1 when $N \rightarrow \infty[15]$.

\section{B. Recall Process with One-to-Many Associations}

Let us discuss the recall process with one-to-many associations. The external input $\tilde{h}_{i}$ of (16) is injected at time $t=-1$ (Model 1) and $h_{i}$ of (17) is injected at time $t=0$ (Model 2).

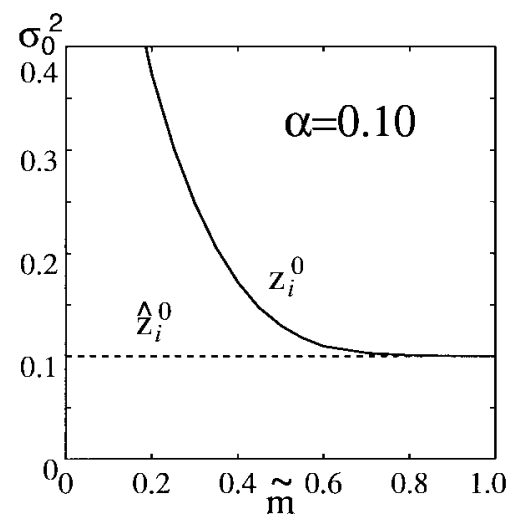

Fig. 3. The initial overlap $\tilde{m}$ and the variance of crosstalk noise $\hat{z}_{i}^{0}$ and $z_{i}^{0}$. Shown is a case where the loading rate is $\alpha=0.1$. The variance $\sigma_{0}^{2}$ approximates to $\alpha$ as $\tilde{m} \rightarrow 1$.

In both cases, the external input is removed at the next time step. We have treated the case where the three associative vectors are associated with the key vector $\left(k^{\mu}=3\right)$, but the present analysis can easily be extended to general cases with arbitrary $k^{\mu}$. In Appendix A, we derived the macroscopic state equations for arbitrary $k^{\mu}$.

First, let us analyze the case where the key input $\boldsymbol{y}$ is the same as the first key vector $\boldsymbol{\eta}^{1}$, namely, $\tilde{m}=1.0$. Fig. 4 shows the temporal evolutions of overlap. The abscissa denotes the time $t$, and the ordinate denotes the overlaps $\tilde{m}=1.0$ of (5) at time $t=-1$ and $m_{t}^{1}$ of (10) at time $t \geq 0$. The curves in each panel represent the results for different values of similarity ( $a=0.0,0.1, \cdots, 1.0$ from bottom to top). The overlaps obtained by Model 1 are in the panels from (a) to (c), and those obtained by Model 2 are in the panels from (d) to (f). For each model, the results obtained by simulations [(a) and (d)], those obtained by theoretical analysis with the firstorder theory [(b) and (e)], and the fourth-order theory [(c) and (f)] are shown. In the simulation studies, the dimensions of the key and the associative vectors were set to $N=M=1000$, namely, $\beta=1.0$, and $p=30$ one-to-three associations were memorized in the networks, that is, $\alpha=0.09$.

As can be seen in the figure, if similarity $a$ is greater than some critical similarity $a_{c}$, the target vector $\boldsymbol{\xi}^{1,1}$ can be recalled successfully. But if $a$ is less than $a_{c}$, the output state converges on the mixture state $\zeta^{1}$ or a spurious state. The critical similarity of Model 1 obtained by simulation is $a_{c}>0.6$, that obtained by the first-order theory is $a_{c}>0.3$, and that obtained by the fourth-order theory is $a_{c}>0.6$. For Model 2, they are $a_{c}>0.3, a_{c}>0.1$, and $a_{c}>0.3$, respectively. Under conditions where $\tilde{m}=1.0, \alpha=0.09$, and $\beta=1.0$ hold, the critical similarity of Model 2 is smaller than that of Model 1. The overlaps obtained by the higher-order theory are in good agreement with those obtained by simulation.

\section{Comparison of Models}

Next, let us discuss which model has smaller critical similarity for various loading rates $\alpha$. First of all, let us consider the case where $\tilde{m}=1.0$ and $\beta=1.0$ hold. As the Appendix shows, the variance $\sigma_{0}^{2}$ at time $t=0$ of (26) is larger than the variance $\tilde{\sigma}^{2}$ at time $t=-1$ of (24), because $U_{0}>0$. Since 


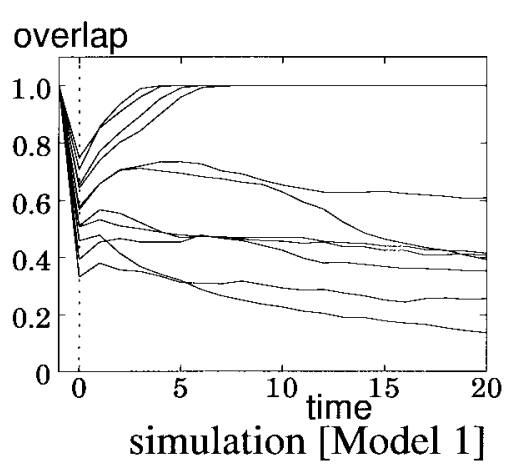

(a)

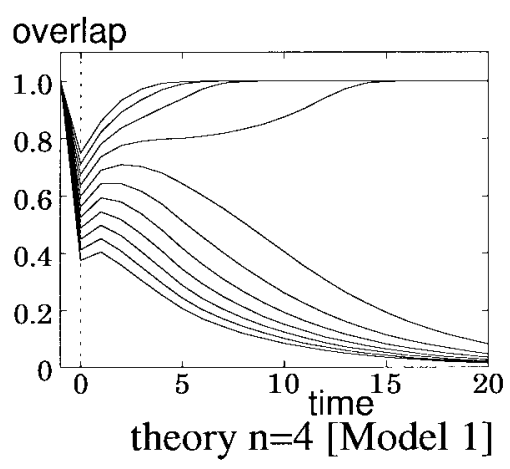

(c)

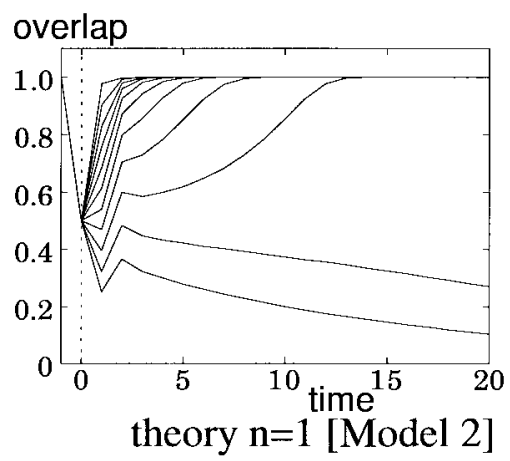

(e)

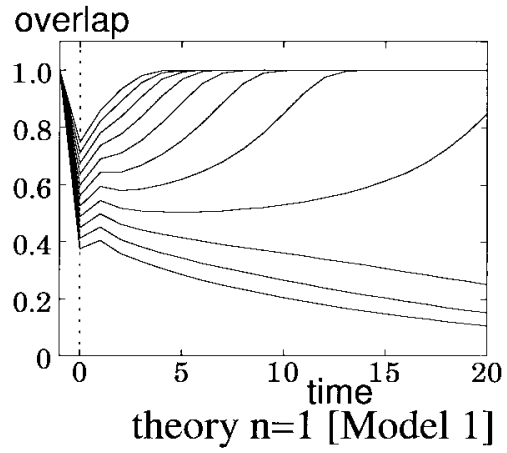

(b)

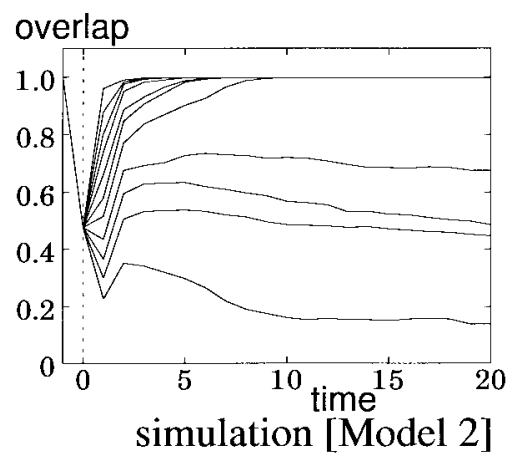

(d)

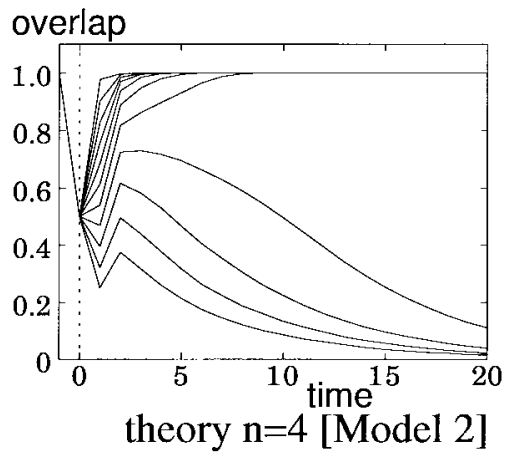

(f)

Fig. 4. Temporal evolutions of overlaps $\tilde{m}$ and $m_{t}^{1}$. Shown is a case where the loading rate is $\alpha=0.09$ and one-to-three associations ( $k^{\mu}=3$ ) are stored. (a) and (d) indicate the results obtained by simulation studies, where $M=N=1000$. (b), (c), (e), and (f) correspond with the first- ( $n=1$ ) and fourth-order $(n=4)$ theories, respectively. (a)-(c) indicate the results for Model 1, and (d)-(f) for Model 2. The curves represent the overlaps for different values of similarity $(a=0.0,0.1, \cdots, 1.0$ from bottom to top).

the target vector $\xi^{1,1}$ tends to be prevented from recalling by the large $\sigma^{2}$, we expect that the target vector of Model 1 can be recalled better. However, Fig. 4 shows that the critical similarity $a_{c}$ of Model 2 is smaller than that of Model 1.

This can be explained by comparing the signal parts of Model 1 at time $t=-1$ and Model 2 at time $t=0$ with the two respective types of external inputs $\tilde{h}_{i}$ and $h_{i}$. The ratio of $m_{0}^{\kappa}$ to similarity $a$ is smaller than that of $\tilde{m}$ to $a$, because $m_{0}^{\kappa} \approx 0.5$ and $\tilde{m}=1.0$ from (13) and (15). Then, the absolute value of the signal part at $t=0$ is smaller than that at $t=-1$. This means that Model 2 is relatively more sensitive to external input than Model 1.

To verify that the above considerations held in general, we calculated the critical similarity $a_{c}$ for various loading rates $\alpha$. The results for Model 1 are in Fig. 5(a) and those for Model 2 are in (b). The key input $y$ is the same as the key vector $\boldsymbol{\eta}^{1}(\tilde{m}=1.0)$. Data points with error bars were obtained by simulation at $M=N=1000(\beta=1.0)$ and the error bars represent standard deviations. Theoretical results obtained by the first-, second-, third-, and fourth-order theories are indicated by the lines. The lines represent the critical similarity $a_{c}$. The results obtained by the higher-order theory are close to the simulation results. The critical similarity $a_{c}$ of Model 2 for various $\alpha$ is smaller than that of Model 1 where $\tilde{m}=1.0$ and $\beta=1.0$ hold. The reason the critical similarity $a_{c}$ decreases at $\alpha \approx 0.03$ (Fig. 5) is because the mixture state, $\operatorname{sgn}\left(\xi_{i}^{1,1}+\xi_{i}^{1,2}+\xi_{i}^{1,3}\right)$, becomes unstable at that point [11]. Next, let us consider the case where $\tilde{m}<1.0$ and $\beta=1.0$ hold, that is, the key input $y$ is an incomplete key vector. Here, since the signal parts $m_{0}^{\kappa} \xi_{i}^{1, \kappa}$ at time $t=0$ are 


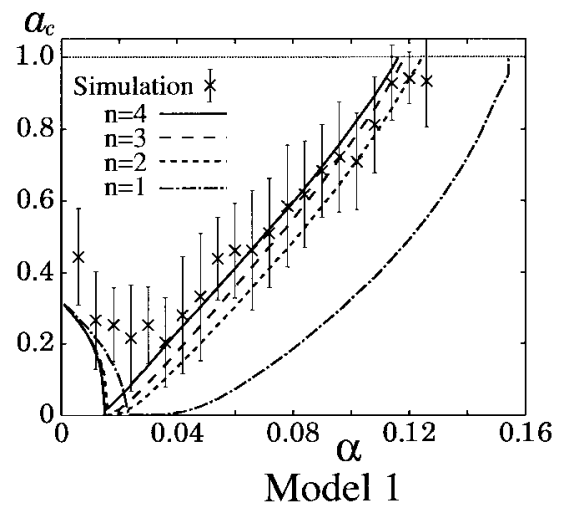

(a)

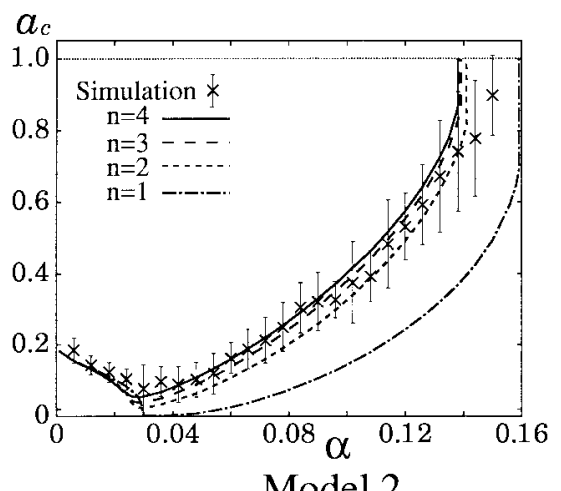

(b)

Fig. 5. The critical similarity $a_{c}$ and the loading rate $\alpha$. The abscissa is the loading rate $\alpha$, and the ordinate is the critical similarity $a_{c}$. (a) indicates the results for Model 1, and (b) for Model 2. The simulation results were obtained by averaging 20 samples, where $\tilde{m}=1.0$ and $M=N=1000(\beta=1.0)$, and the various lines of critical similarity by theoretical analysis indicate the first-, second-, third-, and fourth-order theories.

not always smaller than $\beta \tilde{m} \xi_{i}^{1, \kappa}$ at time $t=-1$ for the small initial overlap, especially for $\tilde{m}<0.5$, the critical similarity between the two models may be different. Fig. 6 shows the critical similarity for the small initial overlap, where $\tilde{m}=0.4$. For all loading rates $\alpha$, the critical similarity of Model 1 is smaller than that of Model 2 where $\tilde{m}=0.4$ in contrast to where $\tilde{m}=1.0$. The critical similarity $a_{c}$ is calculated for various $\tilde{m}$, where $\alpha=0.09$ and $\beta=1.0$, as Fig. 7 shows. The solid line indicates the results for Model 1, and the broken line for Model 2. As the figure shows, the difference between the critical similarity $a_{c}$ of Model 1 and the $a_{c}$ of Model 2 depends on the initial overlap $\tilde{m}$.

In the previous discussions, we considered the case where $\beta=1.0$ holds. The state $x^{0}$ in (6), however, depends on $\beta$. Therefore, we will consider the case where $\beta \neq 1.0$ and $\tilde{m}=1.0$ hold. The critical similarity of Model 1 and that of Model 2 for various values of $\beta$ are in Fig. 8(a) and (b). The critical similarity $a_{c}$ of Model 1 approximately increases with $\beta$, whereas the $a_{c}$ of Model 2 is almost constant for any $\beta$.

Where $\beta=0.5$, the critical similarity of Model 1 becomes smaller than that of Model 2 in contrast to where $\beta=1.0$. The critical similarity, therefore, is calculated for various values of $\beta$, where $\alpha=0.09$ and $\tilde{m}=1.0$, as Fig. 9 shows. As the figure shows, the critical similarity of Model 1 approximately

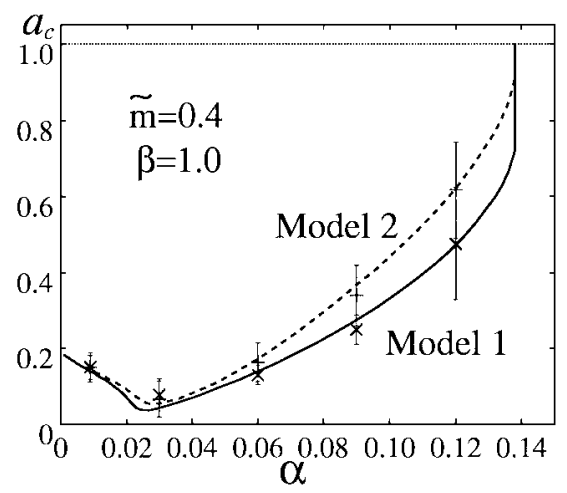

Fig. 6. The critical similarity $a_{c}$ and the loading rate $\alpha$ for the small initial overlap $\tilde{m}=0.4(\beta=1.0)$. The theoretical results were obtained using the fourth-order theory.

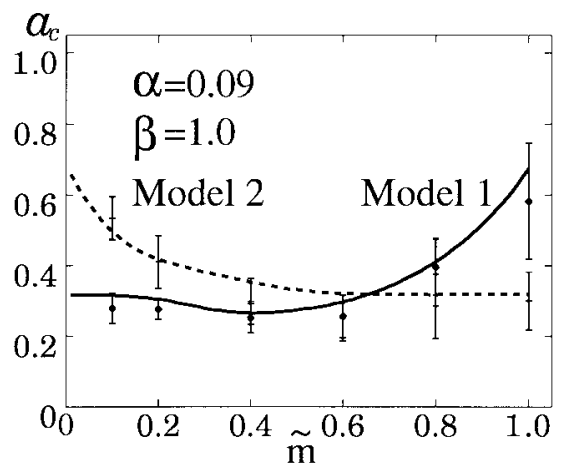

Fig. 7. The critical similarity $a_{c}$ and the initial overlap $\tilde{m}$, where $\alpha=0.09$ and $\beta=1.0$. The theoretical results were obtained using the fourth-order theory.

increases with $\beta$, while that of Model 2 is almost constant as expected from Fig. 8. Comparing the state $\boldsymbol{x}^{0}$ of Model 1 with the state $\boldsymbol{x}^{1}$ of Model 2, let us discuss these $\tilde{m}$ and $\beta$ dependencies in Figs. 7 and 9. The state $x^{0}$ of Model 1 is given by

$$
x_{i}^{0}=\operatorname{sgn}\left(\beta \tilde{m} \xi_{i}^{1,1}+\beta \tilde{m} \xi_{i}^{1,2}+\beta \tilde{m} \xi_{i}^{1,3}+\tilde{z}_{i}+\tilde{h}_{i}\right)
$$

and it explicitly depends on $\beta \tilde{m}$. Obviously, for smaller $\beta \tilde{m}$ the recall process is influenced more by the crosstalk noise $\tilde{z}_{i}$ and the external input $\tilde{h}_{i}$. Therefore, the critical similarity $a_{c}$ of Model 1 increases with $\beta \tilde{m}$. However, the state $\boldsymbol{x}^{0}$ of Model 2 is given by

$$
x_{i}^{0}=\operatorname{sgn}\left(\beta \tilde{m} \xi_{i}^{1,1}+\beta \tilde{m} \xi_{i}^{1,2}+\beta \tilde{m} \xi_{i}^{1,3}+\tilde{z}_{i}\right) .
$$

Since $x_{i}^{0} \simeq \zeta_{i}^{1}$ holds for small variance $\tilde{\sigma}^{2}$, the overlaps $m_{0}^{\kappa}, \kappa=1,2,3$ are approximately equal to $1 / 2$. The next state $\boldsymbol{x}^{1}$ of Model 2 is given by

$$
x_{i}^{1} \simeq \operatorname{sgn}\left(\frac{1}{2} \xi_{i}^{1,1}+\frac{1}{2} \xi_{i}^{1,2}+\frac{1}{2} \xi_{i}^{1,3}+z_{i}^{0}+h_{i}\right) .
$$

Since $m_{0}^{\kappa} \simeq 1 / 2$ hold, the overlaps $m_{0}^{\kappa}$ do not explicitly depend on both $\tilde{m}$ and $\beta$. Therefore, the recall process of Model 2 hardly depends on both $\tilde{m}$ and $\beta$ compared with that of Model 1.

As Fig. 9 shows, the critical similarity of Model 2 is almost constant, but it slightly increases as $\beta$ decreases $(\beta \leq 0.4)$. It also increases as $\tilde{m}$ decreases in Fig. 7 . The reason for this is 


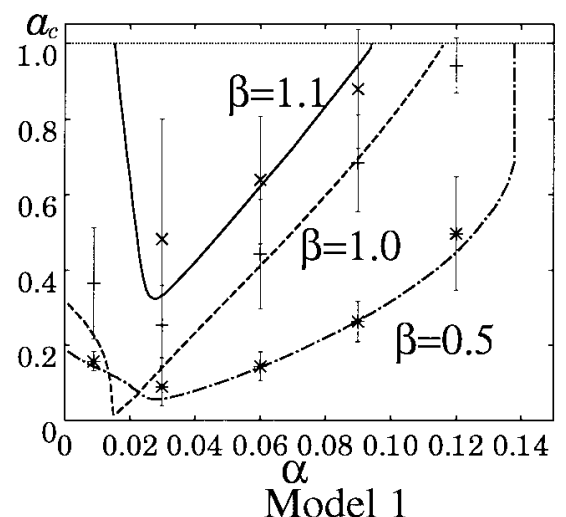

(a)

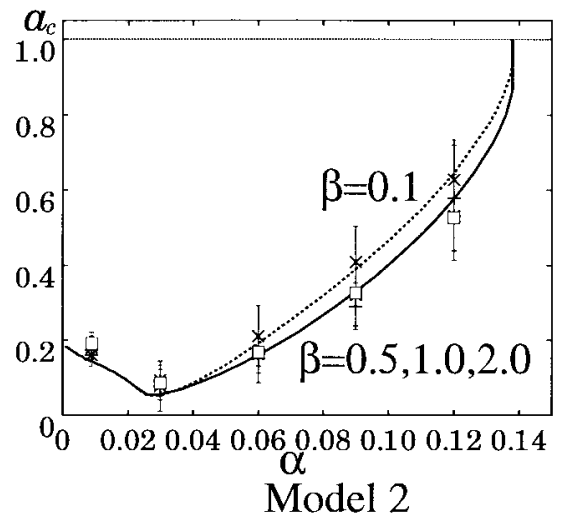

(b)

Fig. 8. The critical similarity $a_{c}$ and the loading rate $\alpha$ for various values of $\beta(\tilde{m}=1.0)$. (a) indicates the results for Model 1, and (b) for Model 2 . The theoretical results were obtained using the fourth-order theory.

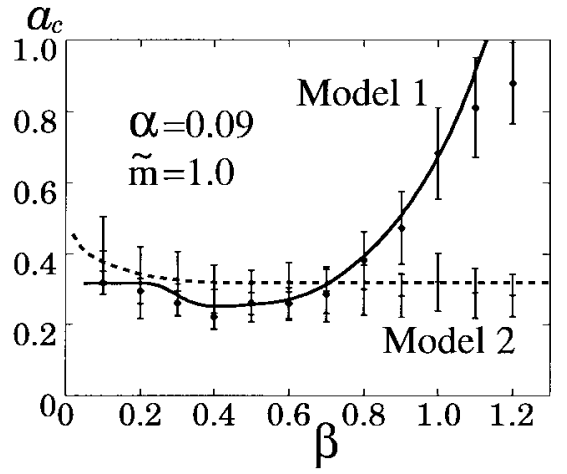

Fig. 9. The critical similarity $a_{c}$ and the dimension ratio $\beta$, where $\alpha=0.09$ and $\tilde{m}=1.0$. The theoretical results were obtained using the fourth-order theory.

as follows. The variance at time $t=0, \sigma_{0}^{2}$, explicitly depends on both $\tilde{m}$ and $\beta$. From (26) and (27), the variance $\sigma_{0}^{2}$ is given by

$$
\begin{aligned}
\sigma_{0}^{2}=\alpha & \frac{2}{\pi} \\
& \cdot\left\langle\exp \left(-\frac{\left(\beta \tilde{m} \xi_{i}^{1,1}+\beta \tilde{m} \xi_{i}^{1,2}+\beta \tilde{m} \xi_{i}^{1,3}\right)^{2}}{2 \alpha \beta}\right)\right\rangle_{(\xi)}^{2}
\end{aligned}
$$

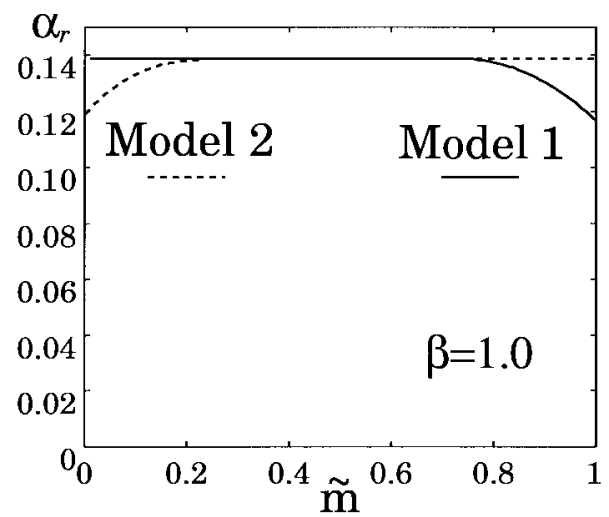

Fig. 10. The critical loading rate $\alpha_{r}$ and $\tilde{m}(\beta=1.0)$. The theoretical results were obtained using the fourth-order theory.

where $\langle\cdot\rangle_{(\xi)}$ stands for the average over the associative vectors $\xi^{1,1}, \xi^{1,2}$, and $\xi^{1,3}$. Since the variance $\sigma_{0}^{2}$ increases as $\beta \tilde{m}$ decreases, the critical similarity $a_{c}$ increases as either $\tilde{m}$ or $\beta$ decreases.

\section{Critical Loading Rate $\alpha_{r}$}

Let us consider vertical lines and intersection points between the critical similarity $a_{c}$ and $a=1$ in Figs. 5, 6, and 8 . They indicate critical value of the loading rate $\alpha$, where the reachable equilibrium states from the initial state by the external input are disappeared. We define the critical value as critical loading rate $\alpha_{r}$ where the reachable equilibrium states are disappeared when the external input with $a=1.0$ is supplied. $\alpha_{r}$ is obviously smaller than the storage capacity $\alpha_{c}=0.138$ of the autoassociative network of the present model. We note that the value of the storage capacity obtained by the first-, second-, third-, and fourth-order theories is $\alpha_{c}=$ $0.160,0.142,0.140,0.139$. Using the higher-order theory, it approaches $\alpha_{c}=0.138$ obtained by the equilibrium theory [10]. In some literatures, $\alpha_{c}$ is also termed "critical loading rate." In the present paper, however, we distinguish the critical loading rate from the storage capacity. We note that the storage capacity $\alpha_{c}$ is defined as the transition point where all equilibrium states become unstable. Since the dynamical behavior must be taken into account in order to evaluate $\alpha_{r}$, it cannot be treated by the equilibrium theory, but by the dynamic theory.

In Fig. 5, the critical loading rate of Model 1 is $\alpha_{r}=0.155,0.125,0.119,0.117$ for first-, second-, third-, and fourth-order theories and that of Model 2 is $\alpha_{r}=0.160,0.142,0.140,0.139 . \alpha_{r}$ of Model 2 is almost equal to the storage capacity $\alpha_{c}=0.138$ of the autoassociative networks, but $\alpha_{r}$ of Model 1 is smaller than $\alpha_{c}$. Comparing Fig. 5(a), (b) with Figs. 6 and 8, we guess that $\alpha_{r}$ of Model 1 strongly depends on $\tilde{m}$ and $\beta$, while $\alpha_{r}$ of Model 2 barely depends on $\tilde{m}$ and $\beta$. To verify our guess, $\alpha_{r}$ for various $\tilde{m}$ and $\beta$ are calculated. Fig. 10 shows the critical loading rate $\alpha_{r}$ for various $\tilde{m}$ obtained by the fourth-order theory. The critical loading rate of Model 1 is $\alpha_{r}=0.139$ for $\tilde{m}<0.77$, while $\alpha_{r}$ decreases for $\tilde{m} \geq 0.77$ as $\tilde{m}$ increases. The number of reachable associative vectors, therefore, is larger in the case where the incomplete input is supplied than the case 


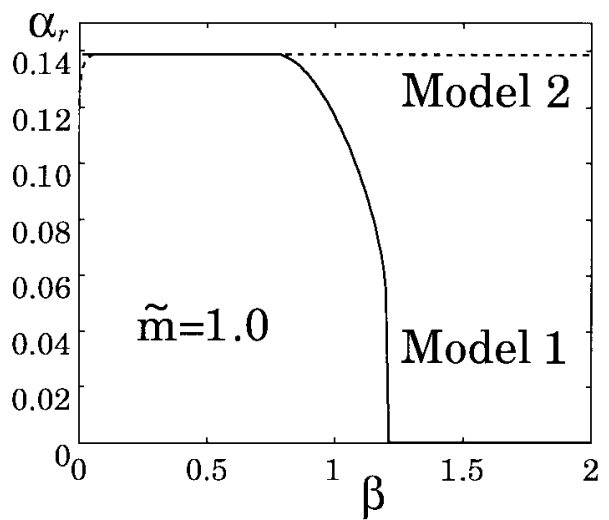

Fig. 11. The critical loading rate $\alpha_{r}$ and $\beta(\tilde{m}=1.0)$. The theoretical results were obtained using the fourth-order theory.

where the complete input is supplied. The critical loading rate of Model 2 is $\alpha_{r}=0.139$ for $\tilde{m} \geq 0.23$, but $\alpha_{r}$ decreases for $\tilde{m}<0.23$. $\alpha_{r}$ of Model 2, therefore, depends on $\tilde{m}$.

Fig. 11 shows the critical loading rate $\alpha_{r}$ for various $\beta$ obtained by the fourth-order theory. The critical loading rate of Model 1 is $\alpha_{r}=0.139$ for $\beta<0.80$, and $\alpha_{r}$ decreases rapidly as $\beta$ increases. The critical loading rate is $\alpha_{r}=0$ for $\beta \geq 1.21$. A critical value $\beta_{c}$ regarding $\beta$, therefore, exist in Model 1. The critical loading rate of Model 2 is $\alpha_{r}=0.139$ for $\beta \geq 0.05$, and $\alpha_{r}$ decrease as $\beta$ decreases. $\alpha_{r}$ of Model 2, therefore, depends on $\beta$ within the small range. Since the critical loading rate $\alpha_{r}$ depends on both $\tilde{m}$ and $\beta, \alpha_{r}$ is calculated for various $\tilde{m}$ and $\beta$. As Fig. 12 shows, the critical loading rate $\alpha_{r}$ becomes one of criteria to compare the models.

Since the storage capacity obtained by the statistical neurodynamics is equal to that obtained by the equilibrium theory [10], [11], [16], it is considered that the embedded associative vectors can be recalled from arbitrary initial state, if the initial state is within the basin of attraction. However, the unreachable states exist in the present model. In other words, above the critical loading rate $\alpha_{r}$, which is smaller than the storage capacity $\alpha_{c}$, all equilibrium states are stable, but cannot be reached by the external input. An interest point is that $\alpha_{r}$ of Model 1 for recalling from the incomplete input is larger than that for recalling from the complete input.

\section{CONCLUSIONS}

We proposed an associative memory model, which can selectively recall one of many associative items by using a cue from context. We discussed the dynamical behavior of the selective recall process in the present model. The model consists of a heteroassociative network and an autoassociative network, which have external input as the context. Since one-to-many associations are stored in the heteroassociative network, many associative items are associated with a single key item. The output, therefore, reaches the mixture state without external input. However, the most suitable associative item (target item) can be selectively recalled by external input. The correlation between the target item and external input is defined as similarity. There is a critical similarity $a_{c}$, above

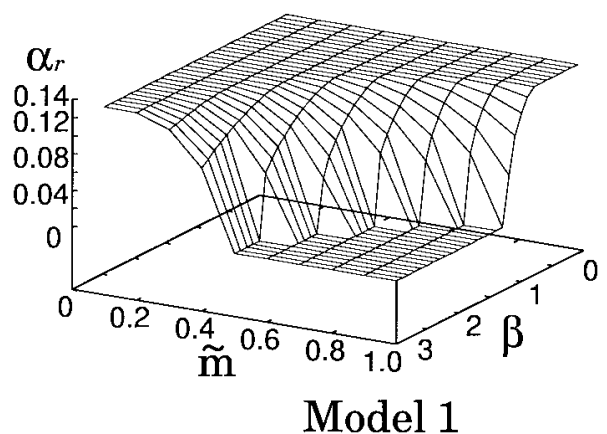

(a)

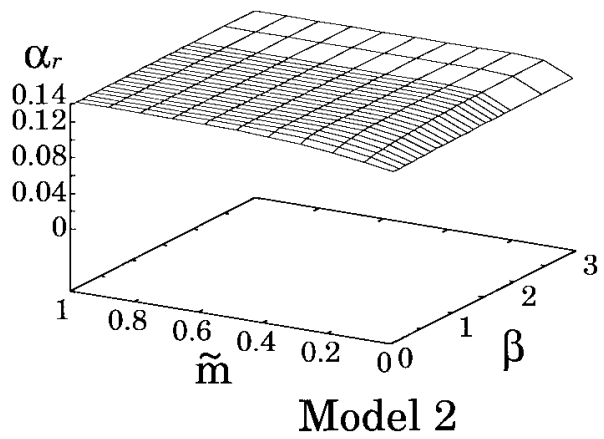

(b)

Fig. 12. The critical loading rate $\alpha_{r}$ (a) indicates the results for Model 1, and (b) for Model 2. The theoretical results were obtained using the fourth-order theory.

which the target vector can be recalled. The main purpose of the present paper is to evaluate the two models using critical similarity.

The influence of external input varies according to the injected time. External input can be injected either into the heteroassociative network or the autoassociative network. We analyzed the dynamics of selective recall for the two models. If the key input is a complete key item or it is sufficiently similar, Model 2, which has external input at time $t=0$, has smaller critical similarity than Model 1, which has external input at time $t=-1$. On the other hand, if the key input is an incomplete key item, Model 1 has smaller critical similarity than Model 2. The difference in the critical similarity between the two models depends on the initial overlap $\tilde{m}$.

The critical similarity of Model 1 increases with $\beta$, which is the dimension ratio of the key vectors to the associative vectors, while that of Model 2 is almost constant as Fig. 9 shows. We discussed why the difference in the critical similarity was changed by the initial overlap $\tilde{m}$ or the dimension ratio $\beta$. We considered states where external input is injected. The state $x^{0}$ explicitly depends on $\beta \tilde{m}$. Therefore, the critical similarity of Model 1 is sensitive to $\beta \tilde{m}$. From (20), the critical similarity of Model 1 increases as $\beta$ or $\tilde{m}$ increases. In Model 2 , the overlaps at time $t=0, m_{0}^{\kappa}$, do not explicitly depend on both $\tilde{m}$ and $\beta$, since $m_{0}^{\kappa} \simeq 1 / 2, \kappa=1,2, \cdots, k$ hold. The reason the critical similarity of Model 2 increases as either $\beta$ or $\tilde{m}$ decreases is because the variance of the crosstalk noise at time $t=0$ depends on both $\tilde{m}$ and $\beta$.

Since the stability of the equilibrium states are not affected by the external input in the present formulation, the storage 
capacity of the present model is $\alpha_{c}=0.138$, which is determined by the equilibrium property of the autoassociative network in the present model. It is considered that the all associative vectors can be recalled from arbitrary initial state which is within the basin of attraction. In the recall process with one-to-many associations, however, we show that there are the unreachable equilibrium states by the external input. That is, above the critical loading rate $\alpha_{r}$ the equilibrium states are not reachable, even if the external input which is equal to the target item is supplied. In order to calculate $\alpha_{r}$, the recall dynamics must be taken into account.

The critical loading rate $\alpha_{r}$ of Model 1 is dependent on both $\tilde{m}$ and $\beta . \alpha_{r}$ decreases as $\tilde{m}$ or $\beta$ increases. The interest points are that $\alpha_{r}$ becomes smaller when the input is more similar to the key item, and that there is a critical value $\beta_{c}$, such that $\alpha_{r}=0$. The value of $\beta_{c}$ is dependent on $\tilde{m}$, i.e., $\beta_{c}=1.21$ for $\tilde{m}=1.0$. The critical loading rate $\alpha_{r}$ of Model 2 is also dependent on both $\tilde{m}$ and $\beta . \alpha_{r}$ decreases as $\tilde{m}$ or $\beta$ decreases.

\section{APPENDIX \\ Macroscopic State EQuations}

The macroscopic state equations of the present model with one-to-many associations are derived from statistical neurodynamics. The theory proposed by Okada [16], which expands on the theory proposed by Amari and Maginu [15], can explain dynamical behavior quantitatively by taking into account the direct correlations between crosstalk noises at different time steps. The first-order equations correspond to the Amari-Maginu theory. We call the cases for $n \geq 2$ higherorder theories. We analyze the general case where key items are associated with different number of associative items.

\section{A. Heteroassociative Network}

From (7), the distribution of crosstalk noise at time $t=-1$ is normally distributed with mean zero and variance

$$
\tilde{\sigma}^{2}=E\left[\tilde{z}_{i}^{2}\right]=\alpha \beta \text {. }
$$

We assume that the distributions at time $t \geq 0$ are normally distributed with mean 0 and variance $\sigma_{t}^{2}$. Note that $z_{i}^{0}$ obeys $\mathcal{N}\left(0, \sigma_{0}^{2}\right)$ rigorously. This assumption is the same as Amari and Maginu's [15] and Okada's [16]. The macroscopic state equations at time $t=0$ are given by

$$
\begin{aligned}
m_{0}^{\kappa} & =\int D z\left\langle\xi^{1, \kappa} \operatorname{sgn}\left(\sum_{\kappa=1}^{k^{1}} \beta \tilde{m} \xi^{1, \kappa}+\tilde{\sigma} z+\tilde{h}\right)\right\rangle \\
\sigma_{0}^{2} & =\alpha+\tilde{\sigma}^{2} U_{0}^{2} \\
U_{0} & =\frac{1}{\tilde{\sigma}} \int D z z\left\langle\operatorname{sgn}\left(\sum_{\kappa=1}^{k^{1}} \beta \tilde{m} \xi^{1, \kappa}+\tilde{\sigma} z+\tilde{h}\right)\right\rangle_{(\xi)}
\end{aligned}
$$

where $\langle\cdot\rangle_{(\xi)}$ stands for the average over the associative vectors $\xi^{1,1}, \xi^{1,2}, \cdots, \xi^{1, k^{1}}$. We define $D z$ as $(2 \pi)^{-1 / 2} \exp \left(-z^{2} / 2\right) d z$.

\section{B. Autoassociative Network}

The macroscopic state equations at time $t \geq 0$ are given by

$$
\begin{aligned}
& m_{t+1}^{\kappa}=\int D z\left\langle\xi^{1, \kappa} \operatorname{sgn}\left(\sum_{\kappa=1}^{k^{1}} m_{t}^{\kappa} \xi^{1, \kappa}+\sigma_{t} z+h \delta_{t, 0}\right)\right\rangle_{(\xi)} \\
& \sigma_{t+1}^{2}=\alpha+U_{t+1}^{2} \sigma_{t}^{2}+2 \alpha \sum_{\tau=t-n+1}^{t} q_{t+1, \tau} \prod_{r=\tau+1}^{t+1} U_{r} \\
& U_{t+1}=\frac{1}{\sigma_{t}} \int D z z\left\langle\operatorname{sgn}\left(\sum_{\kappa=1}^{k^{1}} m_{t}^{\kappa} \xi^{1, \kappa}+\sigma_{t} z+h \delta_{t, 0}\right)\right\rangle_{(\xi)}^{(29)}
\end{aligned}
$$

Note that $\sigma_{-1}$ is defined as $\sigma_{-1} \equiv \tilde{\sigma}$. The correlation between the state at time $t+1$ and that at time $\tau$ is defined as $q_{t+1, \tau} \equiv E\left[x_{i}^{t+1} x_{i}^{\tau}\right]$. We can calculate $q_{t+1, \tau}$ as follows:

$$
\begin{aligned}
& q_{t+1, \tau}=\int D c \int D a \int D b \\
& \left\langle\operatorname{sgn}\left(\sum_{\kappa=1}^{k^{1}} m_{t}^{\kappa} \xi^{1, \kappa}+\sigma_{t}\left(d_{0} a+d_{1} c\right)+h \delta_{t, 0}\right)\right. \\
& \left.\times \operatorname{sgn}\left(\sum_{\kappa=1}^{k^{1}} m_{\tau-1}^{\kappa} \xi^{1, \kappa}+\sigma_{\tau-1}\left(d_{0} b+d_{1} c\right)+h \delta_{\tau-1,0}\right)\right\rangle
\end{aligned}
$$

where $d_{0}=\sqrt{1-d_{1}^{2}}$ and $d_{1}=\sqrt{C_{t, \tau-1} / \sigma_{t} \sigma_{\tau-1}}$. The correlation $q_{t+1,-1}$ is zero.

The correlation $C_{t, \tau-1}$ between the crosstalk noise at time $t$ and that at time $\tau-1$ is given by

$$
\begin{aligned}
& C_{t, \tau-1}= 0, \quad(\tau=t-n+1, n \geq 1) \\
& C_{t, \tau-1}=\alpha q_{t, \tau-1}+U_{t} C_{t-1, \tau-1}, \\
& \quad(\tau=t-n+2, n \geq 2), \\
& C_{t, \tau-1}=\alpha q_{t, \tau-1}+U_{t} U_{\tau-1} C_{t-1, \tau-2}+\alpha \sum_{\eta=\tau-n+1}^{\tau-2} \\
& \cdot q_{t, \eta} \prod_{r=\eta+1}^{\tau-1} U_{r}+\alpha \sum_{\eta=t-n+1}^{t-1} q_{\eta, \tau-1} \prod_{r=\eta+1}^{t} U_{r}, \\
&(t-n+3 \leq \tau \leq t, n \geq 3) .
\end{aligned}
$$

\section{REFERENCES}

[1] D. A. Swinney, "Lexical access during sentence comprehension: (Re)consideration of context effects," J. Verbal Learning and Verbal Behavior, vol. 18, pp. 645-658, 1979.

[2] M. K. Tanenhaus, J. M. Leiman, and M. S. Seidenberg, "Evidence for multiple stages in the processing of ambiguous words in syntactic contexts," J. Verbal Learning and Verbal Behavior, vol. 18, pp. 427-440, 1979 .

[3] W. Kintsch and E. F. Mross, "Context effects in word identification," J. Memory and Language, vol. 24, pp. 336-349, 1985.

[4] Y. Hirai, "A model of human associative processor (HASP)," IEEE Trans. Syst., Man., Cybern., vol. SMC-13, no. 5, pp. 851-857, 1983.

[5] _ "Mutually linked HASPs: A solution for constraint-satisfaction problems by associative processing," IEEE Trans. Syst. Man., Cybern., vol. SMC-15, no. 3, pp. 432-442, 1985. 
[6] Y. Hirai and Q. Ma, "Modeling the process of problem-solving by associative networks capable of improving the performance," Biol. Cybern., vol. 59, pp. 353-365, 1988.

[7] Q. Ma and Y. Hirai, "Modeling the acquisition of counting with an associative network," Biol. Cybern., vol. 61, pp. 271-278, 1989.

[8] D. J. Amit, "Neural networks counting chimes," in Proc. Nat. Academy Sci. USA, vol. 85, pp. 2141-2145, 1988.

[9] I. Guyon, L. Personnaz, J. P. Nadal, and G. Dreyfus, "Storage and retrieval of complex sequences in neural networks," Phys. Rev. A, vol. 38 , no. 12 , pp. 6365-6372, 1988.

[10] D. J. Amit, "Storing infinite numbers of patterns in a spin-grass model of neural networks," Phys. Rev. Lett., vol. 55, no. 14, pp. 1530-1533, 1985.

[11] D. J. Amit, H. Gutfreund, and H. Sompolinsky, "Information storage in neural networks with low levels of activity," Phys. Rev. A, vol. 35, no. 6, pp. 2293-2303, 1987.
[12] J. Hertz, A. Krogh, and R. G. Palmer, Introduction to the Theory of Neural Computation. Reading, MA: Addison-Wesley, 1991.

[13] M. Shiino and T. Fukai, "Self-consistent signal-to-noise analysis and its application to analogue neural networks with asymmetric connections," J. Phys. A: Math. Gen., vol. 25, pp. L375-L381, 1992.

[14] J. J. Hopfield, "Neural networks and physical systems with emergent collective computational abilities," in Proc. Nat. Academy Sci. USA, vol. 79, pp. 2554-2558, 1982.

[15] S. Amari and K. Maginu, "Statistical neurodynamics of associative memory," Neural Networks, vol. 1, pp. 63-73, 1988.

[16] M. Okada, "A hierarchy of macrodynamical equations for associative memory," Neural Networks, vol. 8, no. 6, pp. 833-838, 1995.

[17] M. Kawamura and Y. Hirai, "Storage capacity analysis on a model of human associative processing, HASP," Syst. Comput. Japan, vol. 28, no. 1, pp. 24-33, 1997. 